\section{A case of Mycobacterium goodii infection related to an indwelling catheter placed for the treatment of chronic symptoms attributed to Lyme disease}

\author{
Andrew Shelton, ${ }^{1}$ Luca Giurgea, ${ }^{2}$ \\ Mahdi Moshgriz, ${ }^{3}$ Marc Siegel, ${ }^{4}$ \\ Hana Akselrod ${ }^{4}$ \\ ${ }^{1}$ Department of Internal Medicine, \\ George Washington University School of \\ Medicine and Health Sciences, \\ Washington, DC; ${ }^{2}$ National Institute of \\ Allergy and Infectious Diseases, \\ National Institutes of Health, Bethesda, \\ MD; ${ }^{3}$ Department of Pathology, George \\ Washington University Medical Center, \\ Washington, DC; ${ }^{4}$ Department of \\ Medicine, George Washington \\ University Medical Faculty Associates, \\ Washington, DC, USA
}

\begin{abstract}
Mycobacterium goodii has only rarely been reported to cause invasive disease in humans. Previously reported cases of $M$. goodii infection have included prosthetic joint infections, pacemaker pocket infections, and pneumonia. We present a case of M. goodii bacteremia with concomitant pulmonary septic emboli that developed in a 32-year-old woman with an indwelling central venous catheter (CVC). The CVC had been placed one year previously for intermittent treatment with intravenous, broadspectrum antibiotics, administered by an outside physician for the treatment of symptoms attributed to chronic Lyme disease. Despite our recommendations, the patient declined follow-up in our Infectious Diseases clinic, opting to continue care under her chronic Lyme disease physician. This case clearly demonstrates the potential for serious medical complications that can arise from the inappropriate use of longterm intravenous antibiotics using a CVC to treat non-specific symptoms attributed to Lyme disease and patients should be counseled regarding these risks.
\end{abstract}

\section{Introduction}

Mycobacterium goodii is a rapidly growing non-tuberculous mycobacterium (rNTM) that was identified as a relative of Mycobacterium smegmatis in 1999. It is becoming recognized as an emerging cause of nosocomial infection, particularly in association with surgically implanted materials. ${ }^{1,2}$ However, only rarely have infections with $M$. goodii been associated with CVC infections. We present a case of $M$. goodii cultured from a CVC tip in the setting of persistent $M$. goodii bacteremia and pulmonary septic emboli following longterm exposure to broad-spectrum intravenous antibiotics. This is only the second reported CVC infection due to $M$. goodii, and the first case reported in a patient with extensive outpatient exposure to intravenous broad-spectrum antibiotics.

\section{Case Report}

A 32-year-old woman living in North Carolina but under the care of a local physician in Washington DC for symptoms attributed to chronic Lyme disease presented to George Washington University Hospital with fever, shortness of breath, and increasing confusion. Two years prior to the current presentation, the patient had been given a diagnosis of chronic Lyme disease after she developed multiple symptoms including confusion, photophobia, arthralgia/myalgia, headaches, constipation, and seizures. A computed axial tomography (CT) scan of the chest at the time showed patchy infiltrates and she underwent a bronchoscopy, which did not detect any abnormality including negative acid-fast bacilli smear and culture. Subsequent imaging showed resolution of those infiltrates. She was treated with multiple oral regimens including rifabutin, pyrimethamine, leucovorin, metronidazole and ivermectin. One year prior to her current presentation she had a subclavian CVC implanted for administration of intravenous antibiotics including meropenem, clindamycin, and tigecycline. Most recently, she had completed a three-week course of ciprofloxacin and meropenem every other day, ending several weeks prior to her presentation. After development of her current symptoms, she sought treatment in an emergency department in North Carolina, where she was diagnosed with pneumonia and treated with azithromycin without improvement. She then traveled to Washington, DC for evaluation by her local physician, and subsequently presented to our institution.

In the emergency department the patient was noted to be febrile to $39.5^{\circ} \mathrm{C}$, blood pressure $125 / 73 \mathrm{mmHg}$, heart rate 85 beats/minute. Physical exam revealed fatigued appearance with decreased breath sounds bilaterally, and a CVC with port access in the left chest wall without evidence of infection. Laboratory studies
Correspondence: Andrew Shelton, Department of Internal Medicine, George Washington University School of Medicine and Health Sciences, Ross Hall, 2300 Eye Street, NW, Washington, D.C. 20037, USA. Tel.: +1.703-822-3047 - Fax: +1.828-649-3786. E-mail: ashelton444@gmail.com

Key words: Mycobacterium goodii, post Lyme disease syndrome, catheter-associated bloodstream infection, nontuberculous mycobacteria.

Contributions: AS: conception and design of the work; acquisition, analysis, and interpretation of data for the work; drafting and revision of the work. LG, HA: design of the work; acquisition, analysis, and interpretation of data for the work; drafting and revision of the work. MM, MS: design of the work; acquisition, analysis, and interpretation of data for the work; revision of the work.

Conflict of interest: the authors declare no potential conflict of interest.

Funding: this research was supported by the Intramural Research Program of the NIH, NIAID.

Received for publication: 13 March 2019

Revision received: 25 July 2019.

Accepted for publication: 25 July 2019.

This work is licensed under a Creative Commons Attribution-NonCommercial 4.0 International License (CC BY-NC 4.0).

(C) Copyright: the Author(s), 2019

Licensee PAGEPress, Italy

Infectious Disease Reports 2019; 11:8108

doi:10.4081/idr.2019.8108

revealed leukocytosis (white blood count 11,370 cells $/ \mathrm{mL}, \quad 85 \%$ neutrophils). Contrast CT chest showed bilaterally symmetric opacification with bronchovascular distribution and innumerable pulmonary nodules (Figure 1). Multifocal pneumonia due to septic emboli was suspected, and vancomycin plus piperacillin-tazobactam were started. A trans-thoracic echocardiogram (TTE) found no evidence of valvular vegetations. No other foci of infection were apparent on physical examination. After 72 hours anaerobic blood culture bottles (BACTEC Plus Aerobic/F, Becton Dickinson, Sparks, Maryland) revealed growth of beaded Gram-positive, acid-fast rods suggestive of mycobacterial species.

After lengthy discussion with the patient and her family, the CVC was removed. Culture of initial blood cultures and the CVC tip revealed growth of $M$. goodii. The patient's antibiotics were 
empirically changed to azithromycin 500 mg intravenously daily, ciprofloxacin 400 mg intravenously every 12 hours, and meropenem $1 \mathrm{~g}$ intravenously every 8 hours. The patient clinically improved on this regimen. The patient returned to North Carolina before the identification and sensitivities of the mycobacterial species was available. Therefore, she was empirically switched to an oral regimen of trimethoprim/sulfamethoxazole $800 \mathrm{mg} / 160 \mathrm{mg}$ twice daily, azithromycin $250 \mathrm{mg}$ once daily, and ciprofloxacin $500 \mathrm{mg}$ twice daily for two-weeks from time of line removal. The patient was counseled extensively to avoid future placement of a CVC in the absence of a clear medical indication. Blood cultures drawn after CVC removal were negative at the time of her discharge but subsequent to discharge also grew M. goodii. The patient cancelled her follow-up appointment in our Infectious Diseases clinic and she was lost to follow-up.

The mycobacterial isolate was sent to Quest Diagnostics Labs (San Juan Capistrano, California) for the beta subunit

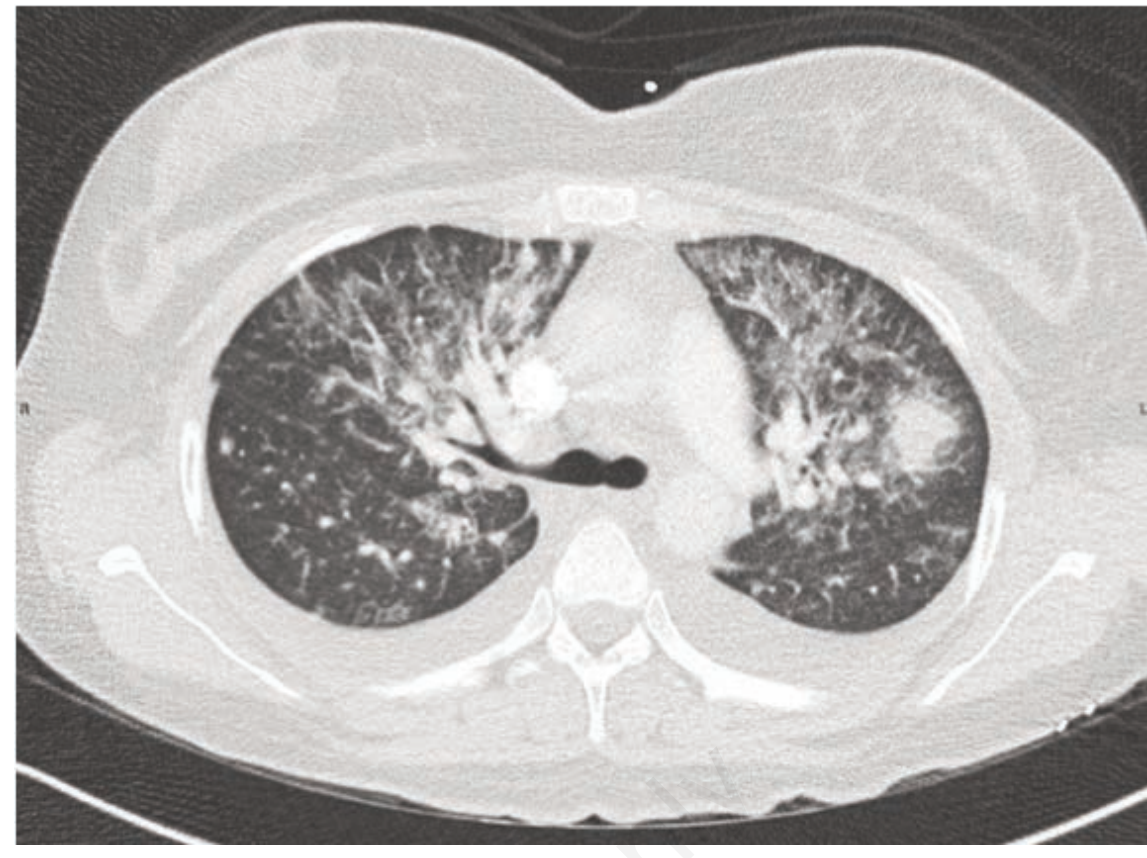

Figure 1. Computed axial tomography of the chest performed with intravenous contrast showing bilateral nodular opacifications in a bronchovascular distribution.

Table 1. Cases of rapid-growing non-tuberculosis mycobacteria (rNTM) identified in blood cultures at our institution, $2012-2018$.

$\begin{array}{lccccccc}\text { Case } & \begin{array}{c}\text { Month/ } \\ \text { Year }\end{array} & \text { Organism } & \begin{array}{c}\text { Central } \\ \text { line }\end{array} & \begin{array}{c}\text { line } \\ \text { removed }\end{array} & \begin{array}{c}\text { Culture } \\ \text { clearance }\end{array} & \text { Antibiotics } & \text { Outcome } \\ 59 \text {-year-old man with ESRD } & 09 / 2018 & \text { M. chelonae } & \text { CVC, tunneled } & \text { Y } & \text { Y } & \begin{array}{c}\text { ciprofloxacin and } \\ \text { metronidazole } \\ \text { for 7 days }\end{array} & \text { Infection resolved }\end{array}$

\begin{tabular}{|c|c|c|c|c|c|c|c|}
\hline $\begin{array}{l}\text { 32-year-old woman with } \\
\text { "Chronic Lyme disease" }\end{array}$ & 03/2018 & M. goodii & CVC, port & Y & $\mathrm{N}$ & See text & Declined follow-up \\
\hline 70-year-old man with & 02/2018 & M. mucogenicum & PICC & $\mathrm{Y}$ & Y & piperacillin/ & Infection \\
\hline Parkinson's Disease & & & & & & tazobactam for 2 weeks & resolved \\
\hline $\begin{array}{l}\text { 28-year-old man with } \\
\text { Crohn's disease }\end{array}$ & $12 / 2017$ & M. mucogenicum & No midline & $\mathrm{Y}$ & Y & linezolid for 2 weeks & Infection resolved \\
\hline $\begin{array}{l}\text { 27-year-old woman with } \\
\text { ESRD }\end{array}$ & $10 / 2016$ & $\begin{array}{l}\text { M. fortuitum } \\
\text { complex }\end{array}$ & CVC, tunneled & $\mathrm{Y}$ & Y & $\begin{array}{l}\text { ciprofloxacin and } \\
\text { clarithromycin, followed by } \\
\text { ciprofloxacin and linezolid } \\
\text { for } 3 \text { months }\end{array}$ & Infection resolved \\
\hline $\begin{array}{l}70 \text {-year-old woman } \\
\text { with Crohn's disease } \\
\text { and recurrent urinary } \\
\text { tract infections }\end{array}$ & $\begin{array}{l}\text { 08/2016 and } \\
\text { 04/2018 }\end{array}$ & $\begin{array}{l}\text { M. fortuitum } \\
\text { complex }\end{array}$ & CVC, PICC & $\mathrm{Y}$ & $\mathrm{Y}^{*}$ & $\begin{array}{l}\text { ciprofloxacin } \\
\text { for } 2 \text { weeks }\end{array}$ & $\begin{array}{l}\text { Cleared initially but } \\
\text { recurred } 1.5 \text { years } \\
\text { later with eventual } \\
\text { clearance after } \\
\text { second PICC line removal }\end{array}$ \\
\hline $\begin{array}{l}\text { 31-year-old woman with } \\
\text { renal transplant }\end{array}$ & 02/2016 & $\begin{array}{l}\text { M. mucogenicum } \\
\text { group }\end{array}$ & CVC, port & Y & Y & $\begin{array}{l}\text { meropenem, } \\
\text { unclear duration }\end{array}$ & Infection resolved \\
\hline $\begin{array}{l}\text { 43-year-old woman } \\
\text { with native-valve } \\
\text { endocarditis }\end{array}$ & $12 / 2015$ & $\begin{array}{l}\text { M. mucogenicum } \\
\text { group }\end{array}$ & PICC & Y & Y & cefepime for 6 weeks & Infection resolved \\
\hline $\begin{array}{l}\text { 52-year-old man with HIV } \\
\text { and recurrent urinary } \\
\text { tract infections }\end{array}$ & 09/2013 & $\begin{array}{l}\text { M. mucogenicum } \\
\text { group }\end{array}$ & PICC & Y & Y & $\begin{array}{c}\text { piperacillin/ } \\
\text { tazobactam and } \\
\text { daptomycin for } 4 \text { weeks }\end{array}$ & Infection resolved \\
\hline $\begin{array}{l}\text { 27-year-old woman with } \\
\text { sickle cell anemia }\end{array}$ & 11/2012 & $\begin{array}{l}\text { M. mucogenicum } \\
\text { group }\end{array}$ & $\begin{array}{l}\text { CVC, internal } \\
\text { jugular }\end{array}$ & Y & Y & $\begin{array}{l}\text { ciprofloxacin } \\
\text { and linezolid for } \\
2 \text { weeks }\end{array}$ & Infection resolved \\
\hline
\end{tabular}

ESRD = end-stage renal disease, $\mathrm{CVC}=$ central venous catheter, $\mathrm{PICC}=$ peripherally inserted central catheter, Midline = peripherally inserted catheter, $\mathrm{Y}=$ blood cultures were drawn after line was removed and were immediately negative, $\mathrm{N}=$ blood cultures were not negative after line removal. *Two instances (see Outcome). 
of RNA polymerase (rpoB) and 16S rDNA sequencing which revealed the organism to be genetically consistent with $M$. goodii with an identity match of $99.86 \%$. Sensitivity testing showed the organism to be resistant to clarithromycin (MIC $>16$ $\mathrm{mcg} / \mathrm{mL}$ ), intermediate to cefoxitin (MIC $32 \mathrm{mcg} / \mathrm{mL}$ ), and susceptible to both ciprofloxacin (MIC $1 \mathrm{mcg} / \mathrm{mL}$ ) and trimethoprim/sulfamethoxazole $0.5 / 9.5 \mathrm{mcg} / \mathrm{mL}$ ).

(MIC

\section{Discussion}

rNTMs are hydrophobic, ubiquitous organisms found in water and soil. They have been observed to form biofilms in non-stagnant water piping, survive a variety of environmental extremes (temperature, $\mathrm{pH}$, scarcity of nutrients), and are regarded as resistant to disinfectants such as chlorine and phenol/glutaraldehyde based substances. ${ }^{3}$ M. goodii has been associated with soft tissue infection, osteomyelitis, bursitis, pneumonia, and achalasia- associated pulmonary disease. ${ }^{1,4,5}$ Cases of $M$. goodil infections have also been reported to complicate a variety of surgical procedures and devices, including; hardware, ${ }^{1,2}$ pacemaker devices, ${ }^{1,6}$ mitral valve annuloplasty, ${ }^{7}$ prosthetic heart valves, ${ }^{8}$ deep brain stimulators, ${ }^{9}$ intra-opthalmic devices, ${ }^{10}$ intra-abdominal mesh, ${ }^{2}$ ventriculoperitoneal shunts, ${ }^{11}$ breast implants, ${ }^{12}$ and intravenous catheters. ${ }^{4}$ In the majority of these cases involving prosthetic material, clearance of the infection was only achieved by removal of foreign material and administration of antibiotic therapy. In only two cases was antibiotic therapy alone successful despite retention of hardware: one involving a deep brain stimulator, ${ }^{11}$ and another a pacemaker pocket infection. ${ }^{6}$

A retrospective review of blood cultures positive for rNTM at our institution over the last six years revealed ten episodes of rNTM bacteremia in nine patients (in addition to the case presented above) summarized in Table 1. These included three cases of $M$. fortuitum complex, one case of $M$. cheloneae, and six cases of M. тисоgenicum. In all cases, a CVC was implicated, and subsequently removed. All cases were treated antibiotics, although the regimen and duration of treatment varied. With the exception of our case, clearance of the bacteremia followed CVC removal. All of the above isolates grew in standard BACTEC blood culture bottles, with subsequent incubation on Löwenstein Jensen media at $36^{\circ} \mathrm{C}$ with $5 \% \mathrm{CO}_{2}$. All specimens were sent to Quest Diagnostics for identification after in-house DNA probes were neg- ative for $M$. tuberculosis complex, $M$. avium complex, and M. kansasii.

Catheter-associated bloodstream infections caused by rNTM are seen most frequently in patients with malignancies that require $\mathrm{CVC}$ for chemotherapy. These infections typically have greater than $90 \%$ clearance rate with catheter removal even in the absence of antibiotic therapy. ${ }^{13,14}$ Unlike the majority of cases of rNTM catheterassociated infections, our patient did not clear her blood cultures immediately after catheter removal. This may have been due to more persistent foci of infection from the septic emboli in her lungs, or possibly due to an infected thrombus at the site of her former long-term CVC. Upper-extremity thrombosis has been described as a risk with the use of catheters similar to that used in our patient, with one study reporting 3 times higher risk for such outcomes. ${ }^{15}$ While mycobacterial native valve endocarditis is exceedingly rare, ${ }^{16}$ and a goodquality TTE was negative in our case, the suspicion of secondary foci of infection warranted close follow-up in this patient, and possible extension of treatment duration for longer than two weeks. Unfortunately, the patient did not return for outpatient follow-up or additional imaging.

Macrolide resistance is a common concern when treating $\mathrm{rNTM},{ }^{17}$ and was documented in our isolate; the patient's history of receiving azithromycin monotherapy prior to her presentation to our hospital may have contributed to this. The empiric multidrug regimen that we started, including the intravenous ciprofloxacin and meropenem while she was an inpatient followed by oral ciprofloxacin, azithromycin, and sulfamethoxazole/trimethoprim as an outpatient, was supported by the susceptibility data and should have been adequate therapy. Due to persistent symptoms, patients with post-Lyme disease syndrome (PLDS) may seek alternative therapies that have limited supporting evidence and can be associated with significant morbidity. Longterm antibiotic therapy has not been shown to have any benefit for PLDS, ${ }^{18}$ but is a clear risk factor for catheter infections and selection of resistant organisms. The use of antibiotics for the treatment of PLDS, especially when given intravenously, has been associated with increased hospitalization, emergency department visits, and infection. ${ }^{19}$ To date, four cases of serious bloodstream infections associated with catheters placed specifically for long-term antibiotic treatment of symptoms attributed to Lyme disease have been reported, with one case resulting in death. ${ }^{20}$ Our patient represents the first case of PLDS treatment complicated by a mycobacterial infection.
As illustrated, the identification and treatment of rNTM organisms can pose particular challenges including multi-drug resistance (especially in patients already exposed to multiple antibiotics), reliance on specialty laboratory methods, and longer times to definitive identification and sensitivity testing. Ultimately, there was a significant trust gap between the patient and our infectious disease physicians, and the patient stated her intention to keep following with her "Lyme-literate" provider and was lost to follow-up.

\section{Conclusions}

Mycobacterial infections, including $M$. goodii, should be considered as potential causes of CVC infections facilitating optimal diagnostic testing and use of appropriate antibiotic regimens. Our patient's CVC and long-term use of antibiotics selected for an unusual biofilm-producing pathogen: $M$. goodii. In contrast to published literature as well as to prior experience with rNTM bloodstream infections at our institution, blood cultures remained positive even after CVC removal and initiation of appropriate antimicrobial therapy, suggesting the presence of a persistent nidus of infection. The patient was counseled on the need for close outpatient follow-up with an infectious disease specialist and avoidance of future long-term line placement without clear medical necessity.

\section{References}

1. Brown BA, Springer B, Steingrube VA, et al. Mycobacterium wolinskyi sp. nov. and mycobacterium goodii sp. nov., two new rapidly growing species related to mycobacterium smegmatis and associated with human wound infections: a cooperative study from the International Working Group on mycobacterial Taxonomy. Int $\mathrm{J}$ Syst Bacteriol 1999;49:1493-511.

2. Ferguson DD, Gershman K, Jensen B, et al. Mycobacterium goodii infections associated with surgical implants at colorado hospital. Emerg Infect Dis 2004; 10:1868-71.

3. De Groote MA, Huitt G. Infections due to rapidly growing mycobacteria. Clin Infect Dis 2006;42:1756-63.

4. Wallace RJ Jr, Nash DR, Tsukamura M, et al. Human disease due to mycobacterium smegmatis. J Infect Dis 1988;158:52-9.

5. Martínez-González D, Franco J, Navarro-Ortega D, et al. Achalasia and 
mycobacterium goodii pulmonary infection. Pediatr Infect Dis J 2011;30:447-8.

6. Marchandin H, Battistella P, Calvet B, et al. Pacemaker surgical site infection caused by mycobacterium goodii. J Med Microbiol 2009;58:517-20.

7. Parikh RB, Grant M. Mycobacterium goodii endocarditis following mitral valve ring annuloplasty. Ann Clin Microb Anti 2017;16:14.

8. Jönsson G, Rydberg J, Sturegård E, Christensson B. A case of Mycobacterium goodii prosthetic valve endocarditis in a non-immunocompromised patient: use of $16 \mathrm{~S}$ rdna analysis for rapid diagnosis. BMC Infect Dis 2012;12:301.

9. Moritz DC, Harrington AT, Slavin K, et al. Deep brain stimulator infection by a novel rapid growing mycobacterium. Acta Neurochir 2017;159:2239-41.

10. Spencer TS, Teske MP, Bernstein PS. Postcataract endophthalmitis caused by mycobacterium goodii. J Cataract Refr Surg 2005;31:1252-3.
11. Uche C, Silibovsky R, Jungkind D, Measley R. Ventriculoperitoneal shuntassociated mycobacterium goodii infection. Infect Dis Clin Pract 2008;16:12930.

12. Pandita A, Thomas S, Granato P, et al. Mycobacterium goodii related breast implant infection: first case and literature review. Clin Microbiol Infect Dis 2017.

13. Helou GE, Hachem R, Viola GM, et al. Management of rapidly growing mycobacterial bacteremia in cancer patients. Clin Infect Dis 2013;56:843-6.

14. Redelman-Sidi G, Sepkowitz KA. Rapidly growing mycobacteria infection in patients with cancer. Clin Infect Dis 2010;51:422-34.

15. Winters JP, Callas PW, Cushman M, et al. Central venous catheters and upper extremity deep vein thrombosis in medical inpatients: the medical inpatients and thrombosis (MITH) study. J Thromb Haemost 2015;13:2155-60.

16. Olalla J, Pombo M, Aguado JM, et al. Mycobacterium fortuitum complex endocarditis - case report and literature review. Clin Microbiol Infect 2002;8:125-9.

17. El Helou G, Viola GM, Hachem R, et al. Rapidly growing mycobacterial bloodstream infections. Lancet Infect Dis 2013;13:166-74.

18. Berende A, ter Hofsede HJ, Vos FJ et al. Randomized Trial of Longer-Term Therapy for Symptoms Attributed to Lyme Disease. N Engl J Med 2016;374:1209-20.

19. Goodlet KJ, Fairman KA. Adverse events associated with antibiotics and intravenous therapies for post-lyme disease syndrome in a commercially insured sample. Clin Infect Dis 2018;67:1568-74.

20. Marzec NS, Nelson C, Waldron PR, et al. Serious bacterial infections acquired during treatment of patients given a diagnosis of chronic lyme disease united states. MMWR Morb Mortal Wkly Rep 2017;66:607-9. 